\title{
A fronto-temporo-parietal network disambiguates potential objects of joint attention
}

2

$4{ }^{1}$ Department of Cognitive Neurology, Hertie Institute for Clinical Brain Research, University of

5 Tübingen, 72076 Tübingen, Germany.

$6{ }^{2}$ Graduate School of Neural and Behavioural Sciences, University of Tübingen, 72074 Tübingen,

7 Germany.

$8 \quad{ }^{3}$ International Max Planck Research School for Cognitive and Systems Neuroscience, University

9 of Tübingen, 72074 Tübingen, Germany.

$10{ }^{4}$ Werner Reichardt Centre for Integrative Neuroscience, University of Tübingen, 72076 Tübingen,

11 Germany.

$12{ }^{5}$ Center for Decision Neuroscience, Faculty of Psychology, University of Basel, 4055 Basel,

13 Switzerland.

$15 \dagger$ These authors contributed equally to this work.

$16 *$ Correspondence to: Peter Thier, Department of Cognitive Neurology, Hertie Institute for Clinical

17 Brain Research, Hoppe-Seyler-Str. 3, 72076 Tübingen, Germany. E-mail: thier@ uni-Tübingen.de. 


\section{Abstract}

22 We use the other's gaze direction to identify her/his object of interest and to shift our attention to

23 the same object, i.e. to establish joint attention. However, gaze direction may not be sufficient to

24 unambiguously identify the object of interest as the other's gaze may hit more than one object. In

25 this case, the observer must use a priori information to disambiguate the object choice. Using

26 fMRI, we suggest that the disambiguation is based on a 3-component network. A first component,

27 the well-known 'gaze following patch' in the posterior STS is activated by gaze following per se.

28 BOLD activity here is determined exclusively by the usage of gaze direction and is independent of

29 the need to disambiguate the relevant object. On the other hand, BOLD activity revealing a priori

30 information for the disambiguation and starting early enough to this end is confined to a patch of

31 cortex at the inferior frontal junction. Finally, BOLD activity reflecting the convergence of both, a

32 priori information and gaze direction, needed to shift attention to a particular object location is

33 confined to the posterior parietal cortex. 


\section{Introduction}

42 We follow the gaze of others to objects of her/his attention and to shift our attention to the same 43 object, thereby establishing joint attention. By associating our object-related intentions, 44 expectations and desires with the other one, joint attention allows us to develop a Theory of (the 45 other's) Mind (TOM). Disposing of a viable TOM is a major basis of successful social interactions

$46 \quad 1,2$ and arguably its absence is at the core of devastating neuropsychiatric diseases such as autism.

47 Human gaze following is geometric ${ }^{3,4}$. This means that we use the other's gaze vector to identify

48 the exact location of the object of interest. The features of the human eye such as the high contrast

49 between the white sclera and dark iris allow us to determine the other's eye direction at high

50 resolution ${ }^{5,6}$. However, knowledge of direction is not sufficient to pinpoint an object in 3D. In

51 principle, differences between the directions of the two eyes, i.e. knowledge of the vergence angle,

52 could be exploited to this end. Yet, this will work only for objects close to the beholder as the angle

53 will become imperceptibly small if the objects are outside the confines of peripersonal space. On

54 the other hand, gaze following remains precise also for objects quite far from the other although

55 the gaze vector will in many cases hit more than one object ${ }^{4}$. Hence, how can these objects be

56 disambiguated? We hypothesized that singling out the relevant object is a consequence of recourse

57 to prior information on the objects and their potential value for the other. For instance, let us assume

58 that the day is hot and that the other's appearance may suggest thirst and the desire to take a sip of

59 something cool. If her/his gaze hit a cool beverage within a set of other objects of little relevance

60 for a thirsty person, the observer might safely infer that the beverage is the object of desire. In this

61 example, gaze following is dependent on prior assumptions about the value of objects for the other.

62 Of course, also the value the object may have for the observer matters. For instance, Liuzza et al.

63 showed that an observer's appetence to follow the other's gaze to portraits of political leaders is 
64 modulated by the degree of political closeness ${ }^{7}$. If the politician attended by the other was a political

65 opponent of the observer, the willingness to follow gaze was significantly reduced. Also knowing

66 that gaze following may be inadequate in a given situation and that the other may become aware

67 of an inadequate behavior will suppress $\mathrm{it}^{8,9}$. However, only assumptions about the object value for

68 the other will help to disambiguate the scene.

69 Following the gaze of others to a particular object is accompanied by a selective BOLD signal in

70 an island of cortex in the posterior superior temporal sulcus (pSTS), the "gaze-following patch

71 (GFP)"'10-12. In these studies, the target object could be identified unambiguously by gaze direction

72 as for a given gaze direction the vector hit one object only. Hence, it remained unclear if the GFP

73 helps to integrate the information needed to disambiguate the object choice in case the gaze vector

74 hits more than one object. In order to address this question, we carried out an fMRI study in which

75 the selection of the object of joint attention required that the observer recoursed on another source

76 of information aside from the gaze cue. 


\section{Results}

Behavioral Performance. Our subjects participated in two fMRI experiments. The first one was a localizer task that allowed us to identify two a priori defined regions of interest (ROI), the GFP and parietal area hLIP (human LIP). To identify the GFP in the temporal lobe, we compared the BOLD activity evoked by following the gaze of a human avatar to one out of 4 possible target objects (gaze following, gf) with the activity evoked by using to avatar's eye color to overtly shift attention to the target sharing this color (color mapping, $\mathrm{cm}$ ). A significant $\mathrm{gf}>\mathrm{cm}$ contrast delineated a region in the pSTS that matched the coordinates of the GFP as known from previous studies ${ }^{11,12}$. Area hLIP was localized by a significant $\mathrm{cm}>b l$ (baseline) contrast in the parietal lobe. The identified region matched values given elsewhere as well ${ }^{13}$. The second experiment was a gaze following task, in which the subjects saw a human avatar gazing along one out of four linearly arranged sets of 3 objects each. The objects were selected from two categories, houses and hands. Hands and houses were distributed such that each category was represented by 1 or 2 exemplars. The observers had to follow the avatar's gaze to a particular object, identified by the conjunction of the avatar's gaze direction and a verbal instruction that specified the object category relevant in a given trial (cf. Fig. 1 for an illustration). After an initial baseline period, during which the avatar looked straight ahead, subjects observed the avatar making a saccade to one of the four object sets. At the same time, the verbal instruction was delivered. It could either be unambiguous ("house" vs. "hand", 1/3 of trials each) or remain uninformative ("none", 1/3 of trials). Depending on the conjunction of gaze direction and instruction three conditions could be distinguished: The unambiguous condition ( $u$; ; the instruction was informative and there was only one of the verbally specified objects in the set), the ambiguous-informative condition (inf; two of the objects were in the set) and the ambiguous-uninformative condition (uninf; the verbal instruction was 
uninformative, i.e. three possible targets). Participants were asked to use the available information to decide on a target and to communicate their decision by making a saccade to that target $5 \mathrm{~s}$ after decision had to consider both gaze direction and the context of the verbal instruction we will refer

In the localizer task, subjects were able to hit targets reliably and without significant difference between the two conditions (median hit rates: $g f: 0.94 \pm 0.13$ s.d.; $\mathrm{cm}$ : $0.92 \pm 0.09$ s.d.; $p=0.6$, two-tailed t-test, $N=19$, Fig. 2). Using the gaze following performance in the localizer task as reference we estimated the following expected hit rates for the contextual gaze following task: 0.94 for the unambiguous condition, $0.94 * 1 / 2$ for the ambiguous-informative and $0.94 * 1 / 3$ for the matched the estimates in the contextual gaze following task very well (comparison by two-tailed ttests, n.s.). This result clearly indicates that the probability to identify an object as a target was exclusively determined by the information provided by gaze direction and verbal instruction and not influenced by biases or uncontrolled strategies.

Task related brain regions. To localize the GFP we contrasted $g f$ with $\mathrm{cm}$ trials of the first experiment. At the group level $(N=19)$ this contrast yielded a patch of significantly larger activity

117 for $g f$ in the pSTS in both hemispheres. The contrast maxima (blue spheres in Fig. 3, upper) were located at $x, y, z=-57,-61,-1$ in the left and at $x, y, z=48,-67,-1$ in the right hemisphere. These locations closely match those known from other studies, visualized as green and cyan spheres for 
tried to localize it in individual subjects by searching for the closest maximum activation which

124 passed a statistical significance threshold ( $p<0.05$, uncorrected) and a cluster size threshold

125 (cluster size $>=6$ voxel). Clusters that lay outside of a sphere with a radius of $10 \mathrm{~mm}$ centered on

126 the group maximum were excluded (proximity criterion). Under these constraints, we were able to

127 determine individual GFPs for nine subjects in the right and for six subjects in the left hemisphere

128 (white spheres ibid., SD of individual locations: right $\mathrm{x}, \mathrm{y}, \mathrm{z}=5,5,3$; left $\mathrm{x}, \mathrm{y}, \mathrm{z}=3,3,5$ ).

An analogous procedure was applied to localize the hLIP using the contrast $\mathrm{cm}>b l$, again based

on trials from the first experiment. The location of maximum activation at the group level was

found to be at $x, y, z=21,-67,50$ (right) and $x, y, z=-21,-67,53$ (left) (blue spheres ibid.) in good

accordance with previous work on saccade related activity in the parietal cortex ${ }^{13}$ (Fig. 3, middle).

The generally much stronger contrast allowed us to determine individual contrast hotspots for all

participants when considering the aforementioned secondary criteria described except for the proximity criterion (white spheres ibid., SD of individual locations: right $\mathrm{x}, \mathrm{y}, \mathrm{z}=4,5,5$; left $\mathrm{x}, \mathrm{y}$,

$\mathrm{z}=4,3,5)$. The latter was not considered because of the wide expanse of significant contrast in

parietal cortex.

In order to identify brain regions specifically activated when the other's gaze is not sufficient to unambiguously single out a target object we ran an exploratory whole-brain analysis. Using the

140 BOLD data from the contextual gaze following experiment, we calculated the BOLD contrast

142 significant $(p<=0.001$, cluster size $>=6$ voxel) for a region in the inferior prefrontal cortex (Fig. 3,

143 bottom) whose group level maxima were found in slightly different locations in the two

144 hemispheres, namely at $x, y, z=-39,11,29$ in the left and $x, y, z=48,20,23$ in the right hemisphere

145 (blue spheres), corresponding to the most lateral part of left BA 8 and the upper right BA 44 . In 15 
subjects we could delineate individual contrast locations that complied with the criterion of a

147 significant activation of at least six adjacent voxel at a threshold of $p=0.05$ (white spheres ibid.,

148 SD of individual locations: right $x, y, z=5,6,6$; left $x, y, z=5,8,6)$. The individual locations

149 scattered around BA 44, BA 8 and BA 9 and henceforth we will refer to this region as the inferior

150 frontal junction (IFJ). In the absence of a priori expectations based on previous studies we did not

151 exclude individual locations that did not match the proximity criterion.

152 Weaker, albeit still significant inf/uninf > $>a$ contrasts were also found in the medial part of left

153 BA 8 at $x, y, z=-3,11,50$, bilaterally in BA 6 at $x, y, z=-21,-4,50$ and $x, y, z=24,-1,50$ and at

$154 x, y, z=36,8,47$ (right hemisphere) not far from the IFJ (cf. Supplementary material Tab. 1).

155 Reversing the contrast, i.e. $u a>$ inf/uninf, we observed bihemispheric significance within BA 13

156 (insula), BA 40, within the cingulate cortex (BA 24 and 31) and within BA 7 (all $p=0.001$, and a

157 minimum of 6 adjacent voxel, cf. Supplementary material Tab. 1). All regions mentioned in the

158 preceding paragraph, even though lighting up in the contrast at the given significance level, did not

159 significantly differentiate between conditions in the following examination of the time courses of

160 the BOLD signals.

Time course of BOLD signals. Successful gaze following in the contextual gaze following task

significant influence of ambiguity suggests that it might play a role in resolving it. In this case, the

we examined the temporal development of BOLD responses associated with the three conditions 
significant individual contrast hot spots, spheres with a radius of $5 \mathrm{~mm}$ were centered at the hot spot coordinates. If this was not the case, instead spheres with a radius of $10 \mathrm{~mm}$, centered at the

171 group level location of the respective contrast were deployed. Fig. 4 depicts the baseline corrected

172 time courses of the BOLD signals averaged across participants, separately for the three conditions

173 and the six ROIs. For all ROIs we found a clear modulation of the BOLD signal by the sequence

174 of trial events with significant activity also in later phases of a trial, independent of condition, with

175 one qualification: the signal evoked in unambiguous trials in the IFJ was weak at best and confined

176 to a short period following the presentation of the cue. On the other hand, in the other two

177 conditions the signal elicited by the cue was not only much stronger but also much more sustained.

178 As anticipated by the activation maps resulting from experiment 1 , the hLIP region showed the

179 overall strongest BOLD signals while those in the GFP and the IFJ were on a lower level. The time

180 course of the BOLD signal in the GFP and the hLIP showed structural similarities. An initial drop

181 after $5 \mathrm{~s}$ was followed by two peaks, one after $10 \mathrm{~s}$ and another after $15 \mathrm{~s}$ (IPS)/16.5 s (GFP). We

182 assume that the first peak is related to the onset of the cue and the second to the go-signal. The

BOLD signal in the IFJ exhibited a qualitatively different shape: the signal appeared to rise in

response to the cue (clearly only for the two ambiguous conditions) but there was no second peak

in relation to the go-signal. To test for significant differences between conditions we performed a

permutation test at each time point (FDR corrected). This test yielded significant differences

between the unambiguous and the ambiguous-uninformative condition between $14 \mathrm{~s}$ and $17 \mathrm{~s}$ in

both hemispheres $(\operatorname{FDR}(p)<0.05)$ and in the IFJ between $10.6 \mathrm{~s}$ and $17 \mathrm{~s}$ (left) and $10.6 \mathrm{~s}$ and 15.4

s (right) $(\operatorname{FDR}(p)<0.05)$ (gray shaded areas in Fig. 4). In other words, the IFJ differentiates earlier 
193 Also, the other areas mentioned in the preceding section on task-related brain areas exhibited

194 BOLD signals that showed a modulation by the sequence of task events. Yet, these profiles did not

195 distinguish between conditions. 


\section{Discussion}

197

198

199

200

201

202

203

204

205

206

207

208

209

210

211

212

213

214

215

216

217

218

This study confirms our previous finding that the GFP in the pSTS plays a major role in processing information on the others' gaze in order to establish joint attention. The present work shows that this role is confined to extracting information on gaze direction. No matter if one or more potential target objects are hit by the gaze vector, the BOLD activity in the GFP is the same. The need to differentiate between objects in case more than one is lying on the gaze vector recruits additional areas that exhibit differential activity. One of these areas, the hLIP in the parietal lobe is also activated in the more traditional, restricted gaze following paradigms, in which the gaze hits one object only. hLIP is necessary for the control of spatial attention ${ }^{14}$. Work on monkey area LIP, arguably homologous to hLIP, has suggested that this area constitutes a priority or saliency map that attracts the "spotlight" of attention to a highlighted map location. The highlighting may be a consequence of bottom-up sensory cues, of symbolic cues or of gaze cues ${ }^{15,16}$. The latter is suggested by single unit recordings from area LIP. Many LIP neurons respond to the appearance of a gaze cue provided the gazed at location lies within the neuron's receptive field ${ }^{17}$. Spatial selectivity for gazed at locations and objects at these locations is also exhibited by many neurons in monkey $\mathrm{GFP}^{18}$. However, unlike neurons in LIP, those in the GFP are selective for gaze direction cueing and do not respond to bottom-up sensory cues highlighting a specific spatial location. This selectivity suggests that the priority map in LIP might draw on input from the GFP. The yoked activation of the hLIP/LIP and the GFP in BOLD imaging studies of gaze following is in principle in accordance with this scenario ${ }^{11,12,17}$. However, the poor temporal resolution of the BOLD signals does not allow us to critically test if the assumed direction of information flow holds true. In any case, bidirectional projections are known to connect monkey area LIP and parts of the STS ${ }^{19}$. One well-established pathway links area LIP and PITd, an area in the lower STS, probably close to the 
219 GFP, known to contribute to the maintenance of sustained attention ${ }^{20,21}$. Yet, the anatomical data 220 available does not allow us to decide if the GFP may indeed be contributing to this fiber bundle.

221 The BOLD signal evoked by gaze following in the hLIP was overall much stronger than in the 222 GFP. Moreover, unlike the GFP signal, it exhibited a clear dependence on the condition. Higher 223 activity was associated with the ambiguous-informative and the ambiguous-uninformative 224 conditions, both associated with unresolved uncertainty as to the correct object. Why should a 225 region thought to coordinate spatial shifts of attention show an influence of target ambiguity, i.e. 226 the need to choose between several potential targets? One possible answer may be that the higher hLIP activity reflects an increased attentional load. More specifically, increased uncertainty in ambiguous trials may have prompted more covert shifts of attention from one object to the other in an attempt to resolve the ambiguity. Although we found no difference in the number of exploratory 230 saccades after the go signal across conditions, we cannot rule out that participants covertly shifted 231 attention between targets in ambiguous trials more than in the other trials and that this might have 232 led to the observed increased activity in the area hLIP. However, a more parsimonious explanation 233 could be that the hLIP constitutes a neural substrate for making decisions under uncertainty 234 independent of the attentional load as suggested by several studies such as ${ }^{22}$.

235 A qualitatively similar dependency on condition also characterized BOLD activity in a region we 236 identified as IFJ based on its location in the frontal lobe at the junction between premotor cortex 237 (BA 6), BA 44 and BA 8. The condition dependency of the IFJ signal is most probably a 238 consequence of the need to shift attention between the two object categories, houses and hands. 239 This interpretation draws on an MEG-fMRI study carried out by Baldauf and Desimone that 240 demanded the allocation of attention to distinct classes of visual objects such as faces and spatial 
241 scenes $^{23}$. Depending on the object of attention, gamma band activity in the IFJ was synchronized

242 either with the fusiform face area (FFA) or the parahippocampal place area (PPA).

243 Hence, the IFJ seems to play a role in allocating attention between objects or object categories and

244 shifting between items. Related work on the putative monkey homologue of human IFJ, the ventral

245 pre-arcuate (VPA), suggests that object representations become highlighted by a match of object

246 templates in VPA and vision-based object representations in inferotemporal cortex ${ }^{24}$. Arguably,

247 the need to choose an object in the ambiguous conditions in our experiment requires a deeper

248 scrutiny of the object options in order to find the match with the object template. This increased

249 effort may be the cause of the stronger IFJ BOLD signal associated with the ambiguous conditions.

250 Within this framework, IFJ can be assumed to highlight specific object representations in

251 inferotemporal cortex. If this was true, information needed by the hLIP to disambiguate the object

252 choice for gaze following would have to be tapped from inferotemporal cortex rather from the IFJ.

253 In sum, our results suggest a fronto-temporo-parietal network for gaze following and the allocation

254 of joint attention underlying the disambiguation of object choices if more than one object is met by

255 the other's gaze vector. Information on the direction of the other's gaze is provided by the GFP,

256 information that allows the hLIP to highlight the spatial positions of all objects lying on the gaze

257 vector. Object-based attention, guided by the IFJ, highlights a relevant object category. The

258 intersection between the two will substantially reduce the possible choices, in most cases singling

259 out just one object that then will become the target of the observer's gaze following response,

260 elicited by the hLIP. 


\section{Methods}

264

265

266

267

268

269

270

271

272

273

274

275

276

277

278

279

280

281

282

283

\section{Participants}

Nineteen healthy, right-handed volunteers (9 females and 10 males, mean age 27.4, s.d. = 3.6) participated in the study over three sessions. Participants gave written consent to the procedures of the experiment. The study was approved by the Ethics Review Board of the Tübingen Medical School and was carried out in accordance with the principles of human research ethics of the Declaration of Helsinki.

\section{Task and procedure}

The study was conducted in three sessions across separate days. On day 1, we instructed participants about the study goals and familiarized them with the experimental paradigms outside the MRI-scanner by carrying out all relevant parts of the fMRI experiments. The following fMRIexperiments included a functional localizer paradigm for the scanning session on day 2 as well as a contextual gaze following paradigm for the scanning session on day 3 .

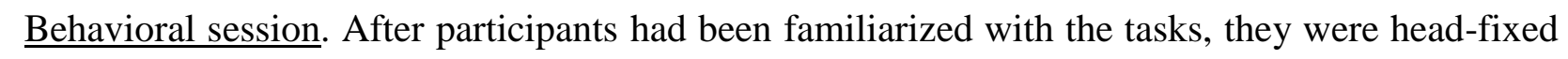
using a chinrest and a strap to fix the forehead to the rest. Subjects were facing towards a frontoparallel screen $($ resolution $=1280 \times 1024$ pixels, $60 \mathrm{~Hz})($ distance to eyes $\approx 600 \mathrm{~mm})$. Eye tracking data were recorded while participants had to complete 80 trials of the localizer paradigm and 72 trials of contextual gaze following.

Localizer task. We resorted to the same paradigm used in ${ }^{11}$, to localize the gaze following network and in particular its core, the GFP. In this paradigm, subjects were asked to make saccades to 
distinct spatial targets based on information provided by a human portrait presented to the observer.

Depending on the instruction, subjects either had to rely on the seen gaze direction to identify the

changing from trial to trial but always mapping to one of the targets, in order to make a saccade to

between the two tasks was the information, subjects had to exploit in order to solve the task, while

the visual stimuli where the same.

This task is associated with higher BOLD activity in the GFP, a region, close to the pSTS, when activation of regions in the intraparietal sulcus (IPS) as well as the frontal cortex that take part in performed 6 runs (40 trials per run) and for reasons of time management during image acquisition, one subject performed 5 runs and two subjects performed 4 runs. angular deg.) image in the center of the screen together with four arrays of drawn objects (houses between the portrait's eyes. After 5 seconds of baseline fixation, the portrait's gaze shifted towards object class of the target (spoken words "hand" or "house") or was not informative ("none"). While 
fixate it until a subsequent blank fixation screen was presented for 8 seconds. The subjects were instructed to perform the task as accurate as possible. They were specifically instructed, when unsure about the actual target, they should still rely on gaze and contextual information and choose the target they believed the avatar to be looking at.

\section{Stimuli}

Control of visual and auditory stimuli as well as data collection was controlled by the Linux based

were specified by varying the distance and the circle radii based on trigonometric calculations. For 
arrays, the circle radii were $335,480,580$ and the distances were 90, 129 and 151 virtual mm. For the $150^{\circ}$ and $210^{\circ}$ arrays, the radii were 380,510 and 590 and the distances were 102,137 and 158 virtual $\mathrm{mm}$. The reason for the difference of radii and distances between $120^{\circ} / 240^{\circ}$ and $150^{\circ} / 210^{\circ}$ arrays was that this allowed to exploit the total width of the screen. This procedure guaranteed that

334 the angle of the gaze vector to all objects on an array was almost identical. This makes it relevant 335 to take contextual information into account in order to choose the true target.

336 The objects were drawings of the two categories houses and hands, downloaded from freely

337 available online sources (http://www.allvectors.com/house-vector/, https://www.freepik.com/freevector/hand-drawn-hands 812824.htm\#term=hands\&page=1\&\%20position=37). The target objects were arranged in four radial directions (three objects in each direction) with the avatar eyes as the origin; in other words, the avatar's gaze always hit one out of three objects along the gaze vector 341 though participants were not able to tell which of the three it was. On each array, either 2 hands 342 and one house or one hand and two houses were present. Further, we fixed the number of hands 343 and houses per hemifield to three. The relative order of the objects was pseudo-randomized from 344 trial to trial.

345 During a trial the participant observed the avatar making a saccade in one of the four directions 346 while simultaneously hearing a verbal instruction providing the additional information by either 347 specifying the target type ("house" or "hand") or being uninformative in that respect ("none") (cf.

348 Fig. 1 for an illustration). In connection with the set of targets specified by the gaze cue the verbal 349 instruction created different levels of ambiguity: unambiguous (only one of the verbally specified 350 types was in the set), ambiguous-informative (two of the types were in the set) and ambiguous351 uninformative (verbal instruction was uninformative, i.e. three possible targets). We created a pool 352 stimulus sets which satisfied three constraints: There was an equal number of trials in which a) the 
353 targets were hands or houses, b) targets were presented with an unambiguous, ambiguous-

354 informative and ambiguous-uninformative instruction, and c) the spatial position (one out of twelve

355 potential positions) of targets was matched. This led to $2 \times 3 \times 12=72$ stimuli sets. We exposed

356 every subject to 180 trials in which each stimulus set was shown twice and for the residual 36 trials,

357 stimuli were drawn from pseudo-randomly from the stimulus pool so that the three criteria above

358 were met.

359 Auditory stimulation was delivered via headphones (Sennheiser HD 201, Wedemark-Wennebostel,

360 Germany, during the behavioral session and the standard air pressure headphones of the scanner

361 system during the MRI sessions). The auditory instructions "hand", "house" and "none" were

362 computer generated with the web application imTranslator (http://imtranslator.net/translate-and-

363 speak/speak/english/) and processed with the software Audacity 2.1.2. The sound files had a

364 duration of $600 \mathrm{~ms}$.

\section{Eye tracking}

367 During all three sessions, we recorded eye movements of the right eyes using commercial eye

368 tracking systems (Behavioral sessions: Chronos Vision C-ETD, Berlin, Germany, sampling rate

$369400 \mathrm{~Hz}$, resolution < $1^{\circ}$ visual angle; Scanning sessions: SMI iView X MRI-LR, Berlin, Germany,

370 sampling rate $=50 \mathrm{~Hz}$, resolution $\approx 1^{\circ}$ visual angle).

371 Eye tracking data was processed as follows. First, we normalized the raw eye tracking signal by

372 dividing it by the average of the time series. Eye blinks were removed using a velocity threshold

373 (>1000\% visual angle). Next, we focused on a time window in which we expected the saccades

374 to the target objects to occur ([go-signal $-500 \mathrm{~ms}$, go-signal $+1800 \mathrm{~ms}])$. Within this time window, 
375 we detected saccades by identifying the time point of maximum eye movement velocity. Pre- and

376 post-saccadic fixation positions were determined by averaging periods of $200 \mathrm{~ms}$ before and after

377 the saccade occurred. Due to partly extensive measurement noise of the eye tracking system, we 378 did not automatize the categorization of the final gaze position. Instead, we plotted X- and Y 379 coordinates of the post-saccadic eye position for every run. An investigator (MG), who was blind 380 to the true gaze target-directions of the stimulus face, manually validated, which trials yielded positions that were clearly assignable to one object location. For the behavioral analysis we only used the valid trials (mean number of valid trials per participant $=80.2$, s.d. $=45.4$, range $=[0,153]$ ) and weighted the individual performance values by its number in order to compute weighted means and SDs. Note, that we used these valid trials only for the behavioral analysis but used all trials of the participants for the fMRI analysis, assuming that eye tracking measurement noise was independent of the performance of the subjects.

\section{fMRI acquisition and preprocessing.}

We acquired MR images using a 3T scanner (Siemens Magnetom Prisma, Erlangen, Germany) with a 20-channel phased array head coil at the Department of Biomedical Magnetic Resonance of the University of Tübingen. The head of the subjects was fixed to the head coil by using plastic foam cushions to avoid head movements. An AutoAlign sequence was used to standardize the alignment of images across sessions and subjects. A high-resolution T1-weighted anatomical scan (MP-RAGE, $176 \times 256 \times 256$ voxel, voxel size $1 \times 1 \times 1 \mathrm{~mm}$ ) and local field maps were acquired. Functional scans were carried out using a T2*-weighted echo-planar multi-banded 2D sequence (multi-band factor $=2, \mathrm{TE}=35 \mathrm{~ms}, \mathrm{TR}=1500 \mathrm{~ms}$, flip angle $=70^{\circ}$ ) which covered the 
For image preprocessing we used the MATLAB SPM12 toolbox (Statistical Parametric Mapping,

399 https://www.fil.ion.ucl.ac.uk/spm/). The anatomical images were segmented and realigned to the

400 SPM T1 template in MNI space. The functional images were realigned to the first image of each

401 respective run, slice-time corrected, coregistered to the anatomical image. Structural and functional

402 images were spatially normalized to MNI space. Finally, functional images were spatially

403 smoothed with a Gaussian kernel (6 mm full-width at half maximum).

\section{fMRI analysis.}

406 We estimated a generalized linear model (GLM) to identify ROIs of single subjects. On these 407 regions, we performed time course analyses to investigate event-related BOLD signal changes. In 408 a first-level analysis, we constructed GLMs for the localizer task $\left(\mathrm{GLM}_{\mathrm{loc}}\right)$ and the contextual gaze 409 following task $\left(\mathrm{GLM}_{\mathrm{cgf}}\right)$. The $\mathrm{GLM}_{\mathrm{loc}}$ included predictors at the onsets of directional cues and of 410 the baseline fixation phase. The $\mathrm{GLM}_{\mathrm{cgf}}$ had predictors at the onset of the contextual instruction. 411 These event specific predictors of both GLMs used the canonical hemodynamic response function 412 of SPM to model the data. We corrected for head motion artifacts by the estimation of six 413 movement parameters with the data of the realignment preprocessing step. Low-frequency drifts 414 were filtered using a high-pass filter (cutoff at $1 / 128 \mathrm{~Hz}$ ).

417 Before collecting the data, we specified the expected locations of two brain areas, hLIP and GFP 418 from fMRI literature. We resorted to the hLIP coordinates of the human homologue of monkey 419 area LIP which had been identified in humans who performed a delayed saccade task ${ }^{13}$. We 
transformed the coordinates into MNI space, using an online transformation method of Lacadie

421 and colleagues ${ }^{27}$ (http://sprout022.sprout.yale.edu/mni2tal/mni2tal.html). ROIs were defined as the

422 voxel of highest signal contrast $\left(\mathrm{GLM}_{\mathrm{loc}}\right.$ : directional cue vs. baseline fixation) the cluster of

423 significant activity (cluster size $\geq 6, \mathrm{p}<0.05$ ) which minimized the spatial distance to the standard

424 coordinates. This contrast has been associated with shifts of attention in response to gaze cues

425 (Marquardt, Ramezanpour et al. 2017). We identified the hLIP regions bilaterally in all 19 subjects

426 with a mean distance of $13.4 \mathrm{~mm}($ s.d. $=3.9 \mathrm{~mm})$ between IPS right $_{\text {and }}$ the standard coordinates and

$42711.93 \mathrm{~mm}(\mathrm{~s} . \mathrm{d} .=3.7 \mathrm{~mm})$ for IPS $_{\text {left. }}$ At the location of the ROI, a sphere (radius $=5 \mathrm{~mm}$ ) was

428 placed.

429 We used a similar procedure for the GFP but with different expected coordinates, a different 430 contrast of the $\left(\mathrm{GLM}_{\text {loc }}\right.$ gaze following vs. color mapping) and the additional constraint that the 431 cluster of significant activity had to be at least partially located within $10 \mathrm{~mm}$ distance around the 432 pSTS standard coordinates. This contrast has been associated to the calculation of the gaze vector 433 direction (for more details see Marquardt et al., 2017). We localized pSTS right in nine individual 434 subjects (mean distance $=6.6 \mathrm{~mm}, \mathrm{s.d} .=3.1 \mathrm{~mm})$ and $\mathrm{pSTS}_{\text {left }}$ in six subjects $($ mean distance $=7.7$

$435 \mathrm{~mm}$; s.d. $=1.4 \mathrm{~mm}$ ). For those subjects and hemispheres where we did not identify pSTS, we 436 reasoned that signal contrast was not high enough and therefore placed a sphere (radius $10 \mathrm{~mm}$ ) at 437 the coordinates obtained from a second level analysis.

\section{Contextual gaze following analysis}

440 We performed an exploratory whole-brain analysis on the data from the contextual gaze following 441 task. We contrasted ambiguous conditions with the unambiguous condition at the group level 
442 (significance threshold $\mathrm{p}<0.001$, cluster size $>=6$ voxel) as well as at the single subject level

443 (significance threshold $p<.05$, cluster size $\geq 6$ voxel). For the single subject analysis, we searched

444 for ROIs that minimized the distance to the group level coordinates. At the identified individual

445 locations (15 subjects) we placed spheres of $5 \mathrm{~mm}$ radius. Again, we used $10 \mathrm{~mm}$ spheres at the

446 group level coordinates for those four subjects for whom we had not identified the ROI in the first

447 level analysis.

448 For every ROI, the mean raw time series of the BOLD signal was extracted using the MATLAB

449 toolbox marsbar 0.44 (http://marsbar.sourceforge.net). The time course of every trial was

450 normalized by the average signal intensity $5 \mathrm{~s}$ before the contextual instruction onset and

451 transformed into \% of signal change. For each participant, we averaged time courses across trials

452 and used the time courses of the three contextual conditions and six ROIs for our analysis. To test

453 differences across conditions for statistical significance, we performed permutation tests at each

454 time point after contextual instruction delivery. To do so we pooled the data of two experimental

455 conditions, respectively, and produced 10,000 random splits for each pool. By computing the

456 differences between the means of these splits, we obtained a distribution of differences under the

457 null hypothesis. Calculating the fraction of values more extreme than the actual difference between

458 means allowed us to obtain a $p$-value for each time bin. To account for the multiple comparison

459 problem, we transformed $p$-values to FDR corrected $q$-values ${ }^{28}$ and considered each time bin with

$460 q<.05$ as statistically significant. 
Acknowledgments: We are grateful to Friedemann Bunjes and Michael Erb for technical support.

464 This work was made possible by a grant from the Deutsche Forschungsgemeinschaft (TH 425/12-

$4652)$

466

467

Authors Contributions: PT developed the conceptual framework of the research. PT, PK and HR

468 designed the experiments. PK and PWD performed the experiments. PK and MG analyzed the data.

469 All authors contributed to the interpretation of results and the writing.

470

471

Competing Interests statement

472 All authors declare to have no competing interests of any sort.

473

474

475

476

477

478

479

480

481 


\section{References}

483

1. Baron-Cohen, S. How to build a baby that can read minds: Cognitive mechanisms in mind reading. Curr. Psychol. Cogn. 13, 513-552 (1994).

2. Baron-Cohen, S. Mindblindness: An essay on autism and theory of mind. (The MIT Press, 1995).

3. Atabaki, A., Marciniak, K., Dicke, P. W. \& Thier, P. Assessing the precision of gaze following using a stereoscopic 3D virtual reality setting. Vision Res. 112, 68-82 (2015).

4. Butterworth, G. \& Jarrett, N. What minds have in common is space: Spatial mechanisms serving joint visual attention in infancy. British journal of developmental psychology 9, 55-72 (1991).

5. Bock, S. W., Dicke, P. W. \& Thier, P. How precise is gaze following in humans? Vision Res. 48, 946957 (2008).

6. Kobayashi, H. \& Kohshima, S. Unique morphology of the human eye. Nature 387, 767-768 (1997).

7. Liuzza, M. T. et al. Follow My Eyes: The Gaze of Politicians Reflexively Captures the Gaze of Ingroup Voters. PLOS ONE 6, e25117 (2011).

8. Teufel, C., Alexis, D. M., Clayton, N. S. \& Davis, G. Mental-state attribution drives rapid, reflexive gaze following. Atten. Percept. Psychophys. 72, 695-705 (2010).

9. Teufel, C. et al. Social Cognition Modulates the Sensory Coding of Observed Gaze Direction. Curr. Biol. 19, 1274-1277 (2009).

10. Laube, I., Kamphuis, S., Dicke, P. W. \& Thier, P. Cortical processing of head- and eye-gaze cues guiding joint social attention. Neuroimage 54, 1643-1653 (2011).

11. Marquardt, K., Ramezanpour, H., Dicke, P. W. \& Thier, P. Following Eye Gaze Activates a Patch in the Posterior Temporal Cortex That Is Not Part of the Human 'Face Patch' System. eNeuro 4, 1-10 (2017). 
12. Materna, S. et al. Dissociable Roles of the Superior Temporal Sulcus and the Intraparietal Sulcus in Joint Attention: A Functional Magnetic Resonance Imaging Study. J. Cogn. Neurosci. 20, 108-119

506 (2008).

13. Sereno, M. I., Pitzalis, S. \& Martinez, A. Mapping of Contralateral Space in Retinotopic Coordinates by a Parietal Cortical Area in Humans. Science 294, 1350-1354 (2001).

14. Corbetta, M. \& Shulman, G. L. Control of goal-directed and stimulus-driven attention in the brain. Nature Reviews Neuroscience 3, 201-215 (2002).

15. Bisley, J. W. \& Goldberg, M. E. Attention, Intention, and Priority in the Parietal Lobe. Annual Review of Neuroscience 33, 1-21 (2010). (2006).

17. Shepherd, S. V., Klein, J. T., Deaner, R. O., Platt, M. L. \& Shepard, R. N. Mirroring of attention by neurons in macaque parietal cortex. Proc. Natl. Acad. Sci. 106, 9489-9494 (2009).

18. Ramezanpour, H., Marciniak, K., Dicke, P. W. \& Thier, P. Neurons in the posterior STS extract facial information for the guidance of gaze following and the establishment of joint attention. (2014).

19. Seltzer, B. \& Pandya, D. N. Parietal, temporal, and occipita projections to cortex of the superior temporal sulcus in the rhesus monkey: A retrograde tracer study. J. Comp. Neurol. 343, 445-463 (1994).

20. Sani, I., McPherson, B. C., Stemmann, H., Pestilli, F. \& Freiwald, W. A. Functionally defined white matter of the macaque monkey brain reveals a dorso-ventral attention network. eLife 8 , e40520 (2019). Cortex. J. Neurosci. 36, 11918-11928 (2016). 
22. Vickery, T. J. \& Jiang, Y. V. Inferior Parietal Lobule Supports Decision Making under Uncertainty in

$528 \quad$ Humans. Cereb Cortex 19, 916-925 (2009).

529 23. Baldauf, D. \& Desimone, R. Neural Mechanisms of Object-Based Attention. Science 344, 424-427

$530 \quad$ (2014).

531 24. Bichot, N. P., Heard, M. T., DeGennaro, E. M. \& Desimone, R. A source for feature based attention in

532 the prefrontal cortex. Neuron 88, 832-844 (2015).

533 25. Benz, P. F. Erstellung einer Software zur partiellen Virtualisierung von Experimentierumgebungen für

534 die perzeptionelle Blickrichtungsbestimmung. (Eberhard Karls Universität, 2008).

535 26. Hübner, C. V. B. Erstellung einer Software zum Design virtueller Experimentumgebungen:

536 Bestimmung des Referenzsystems der visuellen Verarbeitung artifizieller Blicke. (Eberhard Karls

537 Universität, 2008).

538 27. Lacadie, C. M., Fulbright, R. K., Rajeevan, N., Constable, R. T. \& Papademetris, X. More accurate

539 Talairach coordinates for neuroimaging using non-linear registration. Neurolmage 42, 717-725

$540 \quad$ (2008).

541 28. Benjamini, Y. \& Hochberg, Y. Controlling the False Discovery Rate: A Practical and Powerful Approach

542 to Multiple Testing. Journal of the Royal Statistical Society. Series B (Methodological) 57, 289-300

543 (1995).

544

545

546

547 
Baseline fixation

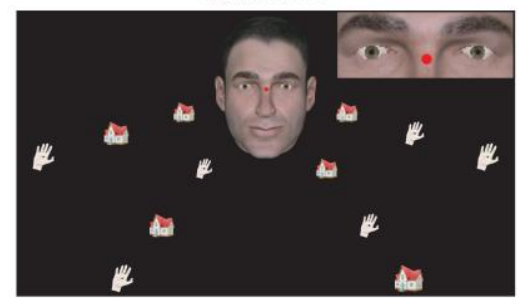

$5 \mathrm{sec}$
Gaze shift + contextual cue

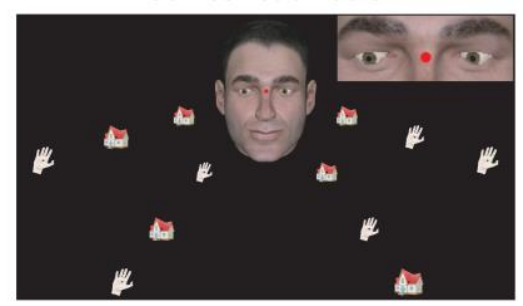

$5 \mathrm{sec}$
Go signal

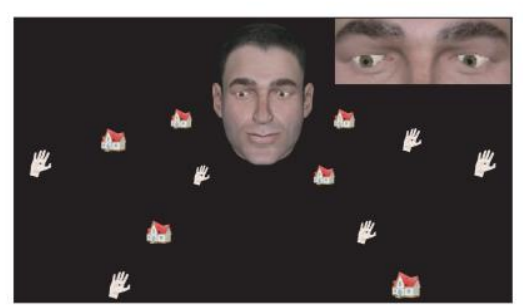

$2 \mathrm{sec}$

550

551 Fig. 1. Contextual gaze following task. An avatar appeared in the center of the screen together

552 with four linearly arranged sets of objects (houses and hands). After a baseline fixation period,

553 the portrait's gaze shifted towards one specific target object simultaneously with an auditory

554 contextual instruction specifying the object class of the target (hand or house) or not, i.e.

555 remaining uninformative ("none"). While maintaining fixation, subjects needed to decide on the

556 target and make a saccade to the chosen target after a go-signal indicated by the disappearance

557 of the fixation dot.

558

559 


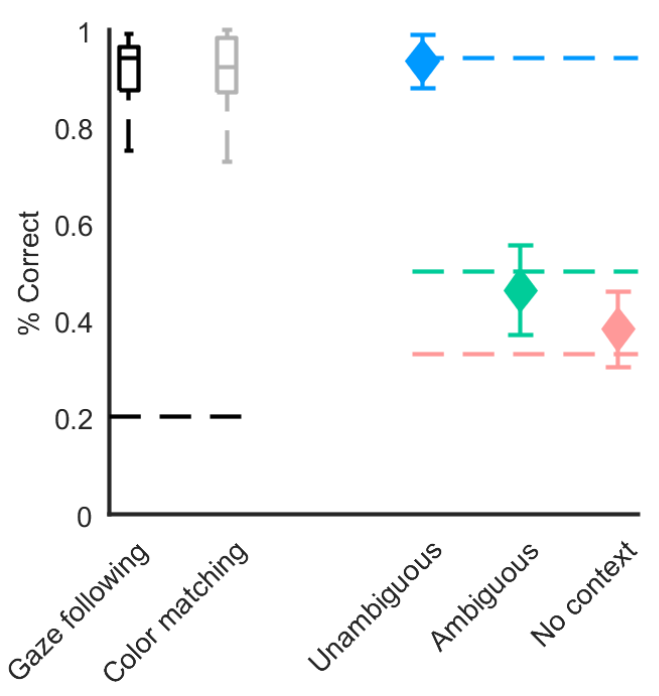

561 Fig. 2. Behavioral performance. Left: Boxplots (black and gray) showing the percentage of 562 correct response in the localizer paradigm (dashed line depicts chance level performance).

563 Right: Plots of correct responses in the contextual gaze following paradigm (weighted mean 564 performance and weighted std, dashed lines depict expected performance). 


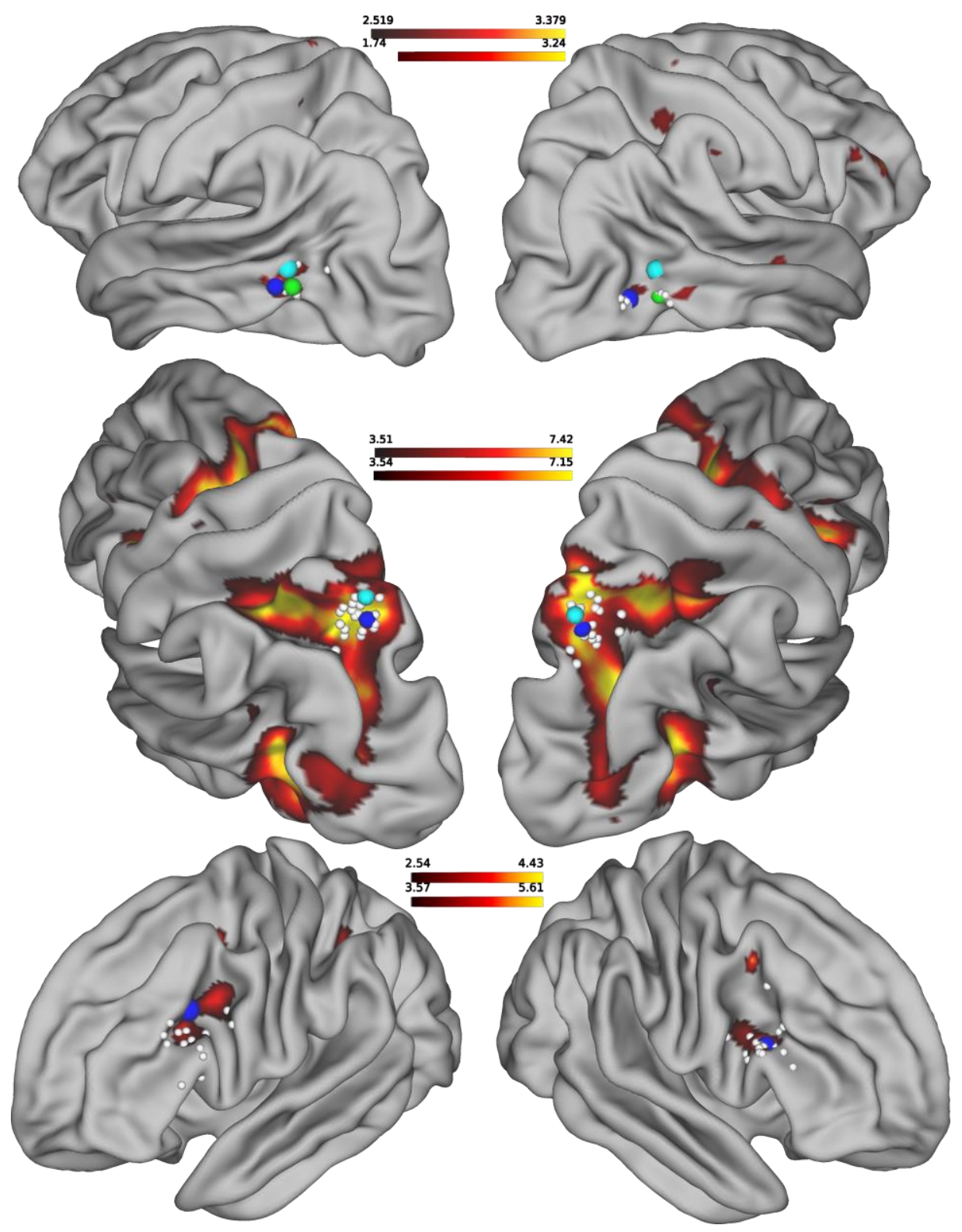

567 Fig. 3. Activation maps. Blue dots mark maximum activation on the group level closest to

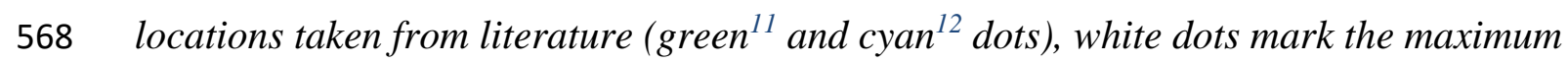

569 activation of those locations which were identifiable on the individual level. Upper row: contrast

$570 \mathrm{gf}>\mathrm{cm}$ (localizer paradigm) used to identify the GFP; Middle row: contrast $\mathrm{cm}>$ bl (localizer

571 paradigm) used to identify saccade-related activity in the hLIP closest to location taken from ${ }^{13}$

572 (cyan dot); Bottom row: uninf > ua (contextual gaze following paradigm). 

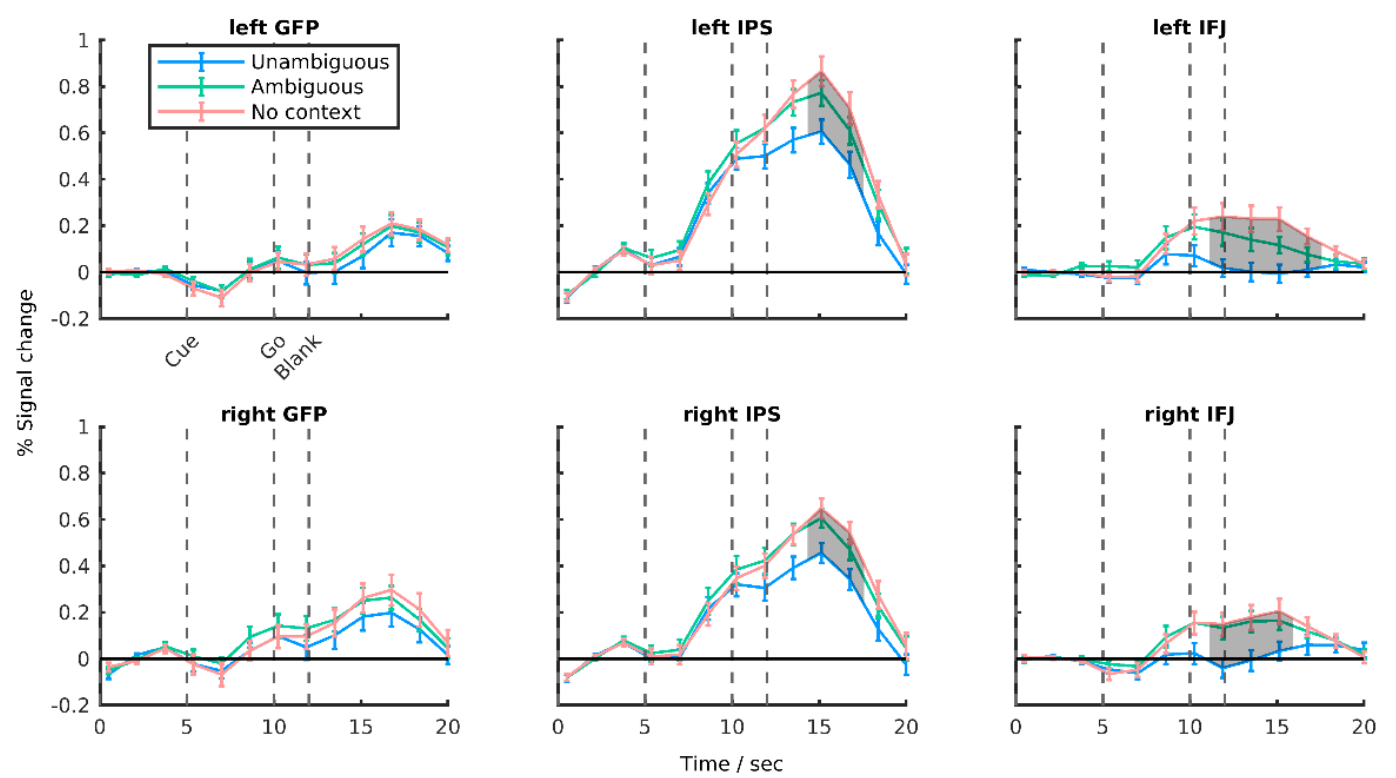

573

$574 \quad$ Fig. 4. Time courses of activation. Time course of mean percent signal change (error bars are

575 SEM). Areas in which conditions showed significant differences are shaded (permutations test,

$576 \quad F D R(p)<0.05)$.

577 


\section{Supplement}

\section{Localizer experiment}

587 As a localizer task we used a cued saccade task, also denoted as a gaze following vs. color mapping $588 \operatorname{task}^{11}$. During a baseline fixation phase, subjects had to fixate on a red dot between the eyes of a 589 photography of a face gazing straight ahead. Below the stimulus face, five colored and horizontally 590 arranged rectangles were presented as gaze targets. After five seconds of baseline fixation, the 591 portrait's eye-gaze shifted towards one of the targets and, simultaneously, its eye color (i.e. the 592 color of the irises) changed to match the color of one of the rectangles. After one second, the red 593 dot disappeared (go signal) and the subjects had to shift their own gaze towards to the correct target 594 and fixate it. There were two different experimental conditions: (1) in gaze following trials, the 595 correct target was determined by the eye-gaze direction of the stimulus face, (2) in color mapping 596 trials, the correct target had the same color as the stimulus irises. The task was performed in several 597 runs, each consisting of four blocks (2 gaze following, 2 color mapping). Each block started with 598 the task instruction as a seven seconds lasting window containing the written words "gaze 599 following" or "color mapping", followed by 10 corresponding trials. Task instruction alternated 600 between blocks. Target objects were counter-balanced such that each rectangle was the target 601 object twice during a block and target order was pseudorandomized. 
Table 1

\begin{tabular}{|c|c|c|c|c|c|}
\hline Corresponding Area & Contrast & $x$ & $y$ & $z$ & Threshold \\
\hline Left-Fusiform (GFP) & \multirow{5}{*}{$g f>c m$} & -57 & -61 & -1 & \multirow{5}{*}{$0.01,6$ Voxel } \\
\hline Right-Fusiform (GFP) & & 48 & -67 & -1 & \\
\hline $\begin{array}{l}\text { Outside defined BAs } \\
\text { (Colliculus) }\end{array}$ & & -6 & -34 & -16 & \\
\hline $\begin{array}{l}\text { Outside defined BAs } \\
\text { (Colliculus) }\end{array}$ & & 9 & -34 & -16 & \\
\hline Right BA45 & & 45 & 32 & 8 & \\
\hline Left BA8 (IFJ) & \multirow{6}{*}{ uninf $>$ ua } & -39 & 11 & 29 & \multirow{6}{*}{$0.001,6$ Voxel } \\
\hline Right BA44 (IFJ) & & 48 & 20 & 23 & \\
\hline Medial BA8 & & -3 & 11 & 50 & \\
\hline Left BA6 & & -21 & -4 & 50 & \\
\hline Right BA6 & & 24 & -1 & 50 & \\
\hline Outside defined BAs & & 36 & 8 & 47 & \\
\hline Left Insula & \multirow{8}{*}{ ua>uninf } & -36 & -16 & 5 & \multirow{8}{*}{$0.001,6$ Voxel } \\
\hline Right Insula & & 42 & -19 & -1 & \\
\hline Left BA40 & & -63 & -28 & 20 & \\
\hline Right BA40 & & 51 & -31 & 17 & \\
\hline Left BA24 (Cingulate cortex) & & -9 & -34 & 44 & \\
\hline Right BA 31 (Cingulate cortex) & & 9 & -16 & 41 & \\
\hline BA7 & & -24 & -43 & 65 & \\
\hline BA7 & & 12 & -46 & 65 & \\
\hline
\end{tabular}

